\title{
Evaluación de la tuna (Opuntia cochenillifera) para la remoción del color en agua potable
}

\author{
Estimation of the nopal cactus (Opuntia cochenillifera) \\ for color removal in drinking water
}

Joaquín Jiménez Antillón'

Maricruz Vargas Camareno

Noemi Quirós Bustos ${ }^{3}$

Fecha de recepción: 20 de enero del 2012

Fecha de aprobación: 19 de marzo del 2012

Jiménez J; Vargas, M; Quirós, N. Evaluación de la

tuna (Opuntia cochenillifera) para la remoción

del color en agua potable.

Tecnología en Marcha. Vol. 25, № 4. Pág 55-62.

I Licenciado en química, máster en Ciencia y Tecnología Ambientales. Centro de Investigación y Protección Ambiental, Escuela de Química, Tecnológico de Costa Rica. Teléfono 2550-2229. Correo electrónico: joajimenez@itcrac.cr

2 Licenciada en química. Centro de Investigación en Protección Ambiental, Escuela de Química, Tecnológico de Costa Rica. Teléfono 2550-2540. Correo electrónico: mvargas@itcrac.cr

3 Bachiller en Química, máster en Gestión Ambiental. Estudiante del doctorado Interuniversitario en Ciencias Naturales para el Desarrollo, del Tecnológico de Costa Rica. Escuela de Química, Tecnológico de Costa Rica. Teléfono 2550-2740. Correo electrónico: nquiros@itcr.ac.cr 


\section{Palabras clave}

Coagulante, floculante, demanda química de oxígeno, río Humo, sustancias húmicas.

\section{Resumen}

El estudio evaluó la eficiencia del cactus de la tuna para remover el color en agua de río y agua artificial para el consumo humano. Se comparó su desempeño con el coagulante sulfato de aluminio y un floculante catiónico. El agua fluvial se caracterizó por presentar alto color, baja turbidez, alcalinidad y dureza y alto porcentaje de oxígeno disuelto.

Las hojas de tuna no mostraron actividad como coagulante único al ser evaluadas en agua de río. Se observó una eficiencia de remoción de color del $94 \%$ en agua artificial, cuando se aplicó como tratamiento único $(45 \mathrm{mg} / \mathrm{l})$, poco superior al tratamiento convencional $\left(20 \mathrm{mg} / \mathrm{l} \mathrm{Al}_{2}\left(\mathrm{SO}_{4}\right)_{3}\right.$ y $2 \mathrm{mg} / \mathrm{l}$ del floculante catiónico) con una remoción del 89\%.

Sin embargo, el agua tratada con tuna dejó un valor de DQO residual del doble $(21 \mathrm{mg} / \mathrm{l})$ con relación al tratamiento convencional. En el tratamiento de agua artificial, la tuna como floculante mostró una remoción de color del $92 \%$ y una DQO residual de 3 I,5 mg/l aplicada a una dosis de $22,5 \mathrm{mg} / \mathrm{l}$ y $7 \mathrm{mg} / \mathrm{l}$ de $\mathrm{Al}_{2}\left(\mathrm{SO}_{4}\right)_{3}$.

La tuna fue poco efectiva para remover el color generado por sustancias húmicas en aguas de baja turbidez y alcalinidad, pero su aplicabilidad como coagulante/floculante en aguas de alta turbidez y alcalinidad es promisoria.

Se recomienda extraer los principios activos de productos naturales para reducir la carga orgánica del agua tratada y evitar la formación de productos secundarios de desinfección.

\section{Key words}

Coagulant, flocculant, chemical oxygen demand, Humo river, humic substances.

\section{Abstract}

The efficiency of nopal cactus to remove color in river and artificial waters for human consumption was evaluated. The performance of nopal was compared with that of aluminum sulfate coagulant and a cationic flocculant. The river water was characterized by high color, low turbidity, alkalinity and hardness, and a high percentage of dissolved oxygen.

The cactus leaves showed no activity as unique coagulant to be evaluated only in river water. In artificial water, as a sole treatment (45 mg/l), an efficiency of $94 \%$ in color removal was observed, slightly higher than that of the conventional treatment $(20 \mathrm{mg} / \mathrm{l}$ $\mathrm{Al}_{2}\left(\mathrm{SO}_{4}\right)_{3}$ and $2 \mathrm{mg} / \mathrm{l}$ of cationic flocculant) with a removal of $89 \%$.

However, water treated with nopal left a residual COD value doubled $(21 \mathrm{mg} / \mathrm{l})$ compared to conventional treatment. In the treatment of artificial water, nopal as a flocculant showed a color removal of $92 \%$ and a residual COD of $31.5 \mathrm{mg} / \mathrm{l}$ applied at a dose of $22.5 \mathrm{mg} / \mathrm{l}$ and $7 \mathrm{mg} / \mathrm{l}$ of $\mathrm{Al}_{2}\left(\mathrm{SO}_{4}\right)_{3}$. The nopal was not very effective to remove the color produced by humic substances in waters of low turbidity and alkalinity, but its applicability as a coagulant/flocculant in waters of high turbidity and alkalinity is promising.

It is recommended to extract the active ingredients of natural products to reduce the organic load of treated water and prevent the formation of disinfection byproducts. 


\section{Introducción}

La demanda de agua potable para la población de la Gran Área Metropolitana (GAM) de Costa Rica ha aumentado dramáticamente en las últimas décadas.

Para hacer frente a esta situación, el Instituto Costarricense de Acueductos y Alcantarillados (AyA) ha buscado nuevas fuentes de abastecimiento de agua, como la captación de 1,8 $\mathrm{m}^{3} / \mathrm{s}$ de agua del embalse El Llano del río Macho (Montero, 2008), un proyecto hidroeléctrico administrado por el Instituto Costarricense de Electricidad (ICE).

Esta fuente de agua proviene de un área cubierta principalmente por bosque primario y recibe tratamiento de potabilización convencional antes de ser enviado al sistema de distribución para su consumo. La población abastecida por esta fuente es de cerca de 600000 habitantes (Gutiérrez, 2009).

La calidad de esta fuente de agua es muy buena, excepto por el color que presenta (Ruiz, 199I). El río Humo es el principal afluente del embalse, con un caudal promedio de $0,7 \mathrm{~m}^{3} / \mathrm{s}$ (Montero, 2008), sin embargo, este río presenta alto color durante todo el año debido a la presencia de sustancias húmicas.

El problema más importante en el agua de este río es su baja turbidez y alcalinidad, además de una alta saturación de oxígeno disuelto, que provoca que el cuerpo de agua libere este gas con el aumento de la temperatura. Lo anterior tiende a dificultar la precipitación de los flóculos formados en el tratamiento.

A su vez, la baja alcalinidad no da oportunidad de formar el hidróxido de aluminio (Montero, 2008), en tanto que la baja turbidez desestimula la adsorción de las moléculas del color (Arboleda, 2000).

La situación arriba expuesta requiere, por tanto, dosis más elevadas de coagulantes y floculantes, que en este caso son el sulfato de aluminio y un polímero catiónico sintético. Ambos productos son importados y constituyen un elevado porcentaje del costo total del agua tratada (Picado, 1984).

Por lo anterior, la utilización de productos naturales como coagulantes o floculantes es de gran interés. Uno de estos productos es la tuna. Martínez et al., (2003) reportan reducciones de turbidez del orden de 80 y $90 \%$ en agua cruda con 20 a 30 FTU, utilizando extractos en metanol y acetato de etilo de la tuna.
Este trabajo resume algunos resultados de un estudio sobre la remoción del color en aguas poco turbias y poco alcalinas como es el caso del río Humo. Se pretende identificar, caracterizar y evaluar la viabilidad técnica de la tuna (Opuntia cochenillifera) como coagulante o floculante para remover el color en agua potable.

\section{Materiales y métodos}

Para evaluar el potencial de la tuna como coagulante o floculante, se realizaron pruebas de jarras utilizando muestras de agua artificial y del río Humo. El agua artificial se preparó adicionando al agua de la cañería caolín y sales de sodio de ácidos húmicos como estándares de turbidez y color, respectivamente. Como alcalinizante se añadió $\mathrm{CaO}$ al 0,3\% en una dosis de I,5 mg/l. Las muestras del río Humo se recolectaron de la toma de agua que administra el ICE, en el Parque Nacional Tapantí.

Para establecer los parámetros óptimos en la remoción del color, tanto en agua artificial como en agua fluvial, se empleó el procedimiento de prueba de jarras recomendado por el Instituto de Salud Colectiva de la Ciudad de Sapporo, Japón (Sapporo City Institute of Public Health, 2003).

Los parámetros del proceso de coagulación fueron: $\mathrm{pH}$ de coagulación, dosis de coagulante y floculante, velocidad y gradiente de velocidad lenta. Posteriormente a los tratamientos se determinaron la turbidez y el color residuales. Se evaluó también la calidad de los flóculos formados según el Índice de Willcomb (Arboleda, 2000).

Para los mejores tratamientos, se cuantificó la demanda química de oxígeno (DQO) del agua filtrada. En ambos tipos de agua se comparó la eficiencia de la tuna como único coagulante versus el $\mathrm{Al}_{2}\left(\mathrm{SO}_{4}\right)_{3}$ al $1 \%$ en conjunto con el polímero catiónico FK-930 S (un polímero del cloruro de dialildimetilamonio) al 0,1\% como floculante. Se comparó también la eficiencia de la tuna como asistente de coagulación (floculante) del sulfato de aluminio con el tratamiento convencional, solamente en muestras de agua artificial.

En todos los ensayos se utilizaron hojas frescas de tuna a las cuales se les eliminó la cáscara, dejando la parte sólida y gelatinosa. Se mezcló la parte sólida y gelatinosa en agua destilada y se agitó lentamente 
durante una hora, se dejó reposar 30 minutos y se filtró por gravedad con un filtro de tela para eliminar partículas.

Para estimar la concentración de la disolución resultante, se determinó el contenido de humedad de la parte gelatinosa y sólida de la planta desecándola a $60^{\circ} \mathrm{C}$ hasta lograr peso constante. El contenido de humedad fue del $91 \%$ y la concentración de la disolución de trabajo de la tuna de 22,5 mg/ml. La caracterización de las aguas se hizo utilizando métodos patrón (APHA, AWWA, WEP, 2005).

\section{Resultados y discusión}

Evaluación de la tuna como coagulante en comparación con el tratamiento convencional

\section{Muestras de agua de río}

Se encontró que las muestras de agua de la época lluviosa presentaron baja alcalinidad (4-24 mg $\left.\mathrm{CaCO}_{3} / \mathrm{l}\right)$ y baja dureza (9-14 mg $\left.\mathrm{CaCO}_{3} / \mathrm{l}\right)$, con porcentajes de saturación de oxígeno disuelto cercanos al 100\%; lo anterior, junto con valores altos de color (85-475 UC) con baja turbidez (9-64 FTU), provocaron difíciles condiciones para remover el color.

Las muestras de la época seca presentaron condiciones desfavorables con relación a la lluviosa, porque fueron menos turbias (I I-25 FTU), de alcalinidad inferior a 1 I mg/l, una dureza inferior a $20 \mathrm{mg} / \mathrm{l}$ pero manteniéndose alto el color aparente ( $142-195$ UC).

Lo anterior justifica la ausencia de actividad coagulante de la tuna (véase cuadro I) al ser aplicada como coagulante único y porque, además, las moléculas con propiedades coagulantes presentes en la tuna poseen menor densidad de carga en comparación con las especies cargadas del aluminio, según lo establece la ley de Schulze Hardy, donde el efecto desestabilizador de los coloides se incrementa conforme mayor sea la carga del contraion añadido (Arboleda, 2000).

El tratamiento convencional óptimo requirió una dosis elevada de coagulante $(30 \mathrm{mg} / \mathrm{l})$ con una dosis normal de floculante $(1 \mathrm{mg} / \mathrm{l})$. Esta elevada dosis se justifica por la baja alcalinidad, dureza y turbidez en presencia de alto color del agua. El elevado valor de DQO del agua filtrada $(6 \mathrm{l} \mathrm{mg/l})$ para el tratamiento óptimo evidencia que el tratamiento convencional está diseñado para clarificar la turbidez pero no la materia orgánica natural (Eikebrokk, 1999).

\section{Muestras de agua artificial}

Se utilizó agua artificial con los siguientes parámetros de calidad inicial: turbidez = 18-22 FTU; color aparente $=150-165 \mathrm{UC} ; \mathrm{pH}=7,06-7,56$; dosis adicional de $\mathrm{CaO}=1,5 \mathrm{mg} / \mathrm{l}$.

En este caso, el desempeño de la tuna ( $94 \%$ de remoción de color) fue mejor que el tratamiento convencional (89\%, véase cuadro 2), resultando un agua tratada con turbidez y color por debajo de los límites de la legislación local de I-5 FTU y 5- I 5 UC, respectivamente (MINSA, 2005) al aplicarse la dosis mínima de 45 mg/l (figura I).

El agua de la cañería presentó una mayor concentración de iones divalentes, comparada con la del río, que junto con los iones $\mathrm{Ca}^{2+}$ añadidos artificialmente, favoreció la desestabilización de los coloides causantes del color y mejoró la capacidad de la tuna para formar flóculos de excelente calidad ( 10 unidades de Willcomb, ver figura I) a bajas concentraciones.

\section{Cuadro I. Calidad del agua del río Humo después de los tratamientos}

\begin{tabular}{|c|c|c|c|c|c|c|c|c|}
\hline Coagulante & $\begin{array}{c}\text { Dosis } \\
\text { coagulante } \\
(\mathrm{mg} / \mathrm{l})\end{array}$ & $\begin{array}{l}\text { Dosis } \\
\text { floculante } \\
(\mathrm{mg} / \mathrm{l})\end{array}$ & $\begin{array}{l}\text { Turbidez } \\
\text { residual } \\
\text { (FTU) }\end{array}$ & $\begin{array}{l}\text { Color } \\
\text { residual } \\
\text { (UC) }\end{array}$ & $\begin{array}{l}\text { Remoción } \\
\text { turbidez (\%) }\end{array}$ & $\begin{array}{l}\text { Remoción } \\
\text { color (\%) }\end{array}$ & $\begin{array}{l}\text { DQO } \\
\text { soluble } \\
(\mathrm{mg} / \mathrm{l})\end{array}$ & $\begin{array}{l}\text { Calidad } \\
\text { flóculo }\end{array}$ \\
\hline $\begin{array}{l}\mathrm{Al}_{2}\left(\mathrm{SO}_{4}\right)_{3}+ \\
\text { floculante KF } \\
930-\mathrm{S}\end{array}$ & 30 & 1,0 & 1 & 12 & 93 & 89 & 61 & 10 \\
\hline Tuna & $45-180$ & 0 & 21 & 125 & 0 & 0 & $\mathrm{NC}$ & 0 \\
\hline
\end{tabular}

$\mathrm{NC}=$ no cuantificado 
Cuadro 2. Calidad del agua artificial tratada con el tratamiento convencional y la tuna como único tratamiento

\begin{tabular}{|l|c|c|c|c|c|c|c|c|}
\hline Coagulante & $\begin{array}{c}\text { Dosis } \\
\text { coagulante } \\
(\mathrm{mg} / \mathrm{l})\end{array}$ & $\begin{array}{c}\text { Dosis } \\
\text { floculante } \\
(\mathrm{mg} / \mathrm{l})\end{array}$ & $\begin{array}{c}\text { Turbidez } \\
\text { residual } \\
(\mathrm{FTU})\end{array}$ & $\begin{array}{c}\text { Color } \\
\text { residual } \\
(\mathrm{UC})\end{array}$ & $\begin{array}{c}\text { Remoción } \\
\text { turbidez } \\
(\%)\end{array}$ & $\begin{array}{c}\text { Remoción } \\
\text { color }(\%)\end{array}$ & $\begin{array}{c}\text { DQO } \\
\text { soluble } \\
(\mathrm{mg} / \mathrm{l})\end{array}$ & $\begin{array}{c}\text { Calidad } \\
\text { flóculo }\end{array}$ \\
\hline $\begin{array}{l}\mathrm{Al}_{2}\left(\mathrm{SO}_{4}\right)_{3}+ \\
\text { floculante KF } \\
930-\mathrm{S}\end{array}$ & 20 & 2,0 & 2 & 16 & 90 & 89 & 10,3 & 6 \\
\hline $\begin{array}{l}\text { Tuna } \\
\text { Tuna }\end{array}$ & 45 & 0 & 0 & 9 & 100 & 94 & 21 & 10 \\
\hline
\end{tabular}

La adición de disolución saturada de $\mathrm{CaO}$, que retuvo cierta naturaleza particulada, suministró sitios donde se indujo la nucleación para el desarrollo de flóculos, los cuales, una vez presentes, actuaron como sitios de adsorción para la materia orgánica natural soluble, que finalmente facilitó la coagulación (Gregor et al., 1997) (Eikebrokk, 1999). Se observó el restablecimiento del sistema coloidal a dosis superiores a los $100 \mathrm{mg} / \mathrm{l}$, por el incremento en la turbidez resultante (figura I).

Martínez reporta reducciones de turbidez del orden de 80 y $90 \%$ en agua cruda con 20 y 30 UNT y 0,53 $\mathrm{mg} / \mathrm{l}$ de $\mathrm{CaCO}_{3}$, utilizando extractos en metanol y acetato de etilo de la tuna (Martínez et al., 2003) donde se obtuvieron dosis óptimas inferiores (de 10 a $20 \mathrm{mg} / \mathrm{l})$ a las de este estudio $(45 \mathrm{mg} / \mathrm{l})$.
Sin embargo, esta autora únicamente ensayó la clarificación de la turbidez que, por su mayor tamaño de partícula, requirió menores dosis de coagulante en comparación con la que se necesita para remover el color (Arboleda, 2000).

El valor de la DQO soluble del agua tratada con tuna mostró el doble del valor del tratamiento convencional y demostró la presencia de sustancias orgánicas oxidables, sin propiedades coagulantes, en el agua tratada.

\section{Evaluación de la tuna como floculante en comparación con el tratamiento convencional}

La tuna como ayudante de coagulación del sulfato de aluminio se ensayó en dos tipos de agua: agua artificial con alcalinidad adicional y agua de río con alcalinidad adicional.

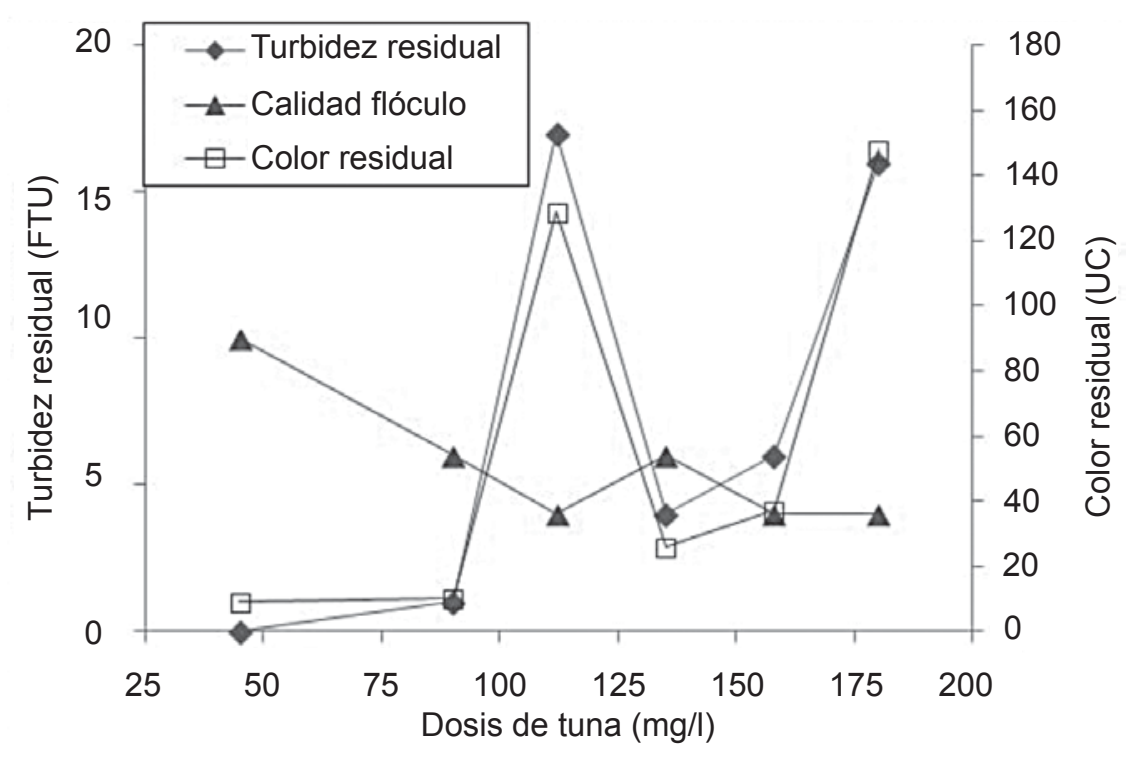

Figura I. Color y turbidez residuales utilizando tuna como coagulante único en agua modelo. 


\section{Muestras de agua artificial}

En este caso, se utilizó agua artificial con las siguientes características: turbidez 21 FTU, color aparente $=136$ UC; $\mathrm{pH}=7,58$ y una dosis de $\mathrm{CaO}=$ I,5 mg/l.

En la figura 2 se observa que con la dosis mínima aplicada de la tuna $(22,5 \mathrm{mg} / \mathrm{l})$ se obtuvo el mejor tratamiento en remoción de color (92\%), que fue levemente superior al tratamiento con solo sulfato de aluminio $\left(7 \mathrm{mg} / \mathrm{l}\right.$ de $\mathrm{Al}_{2}\left(\mathrm{SO}_{4}\right)_{3}$ con una eficiencia del $86 \%$ ) y al tratamiento convencional $(20 \mathrm{mg} / \mathrm{l}$ en $\mathrm{Al}_{2}\left(\mathrm{SO}_{4}\right)_{3}, 2 \mathrm{mg} / \mathrm{l}$ del floculante comercial y una eficiencia del 89\%), (véase el cuadro 3).

Nuevamente, la calidad del agua con tuna estuvo de conformidad con la norma (MINSA, 2005). Pero la DQO del agua tratada $(31,5 \mathrm{mg} / \mathrm{l})$ fue tres veces superior a la del tratamiento convencional (véase el cuadro 3 y la figura 3). De la figura 3 se observa que con una dosis superior de tuna $(67 \mathrm{mg} / \mathrm{l})$ se obtuvo la mejor remoción de materia orgánica soluble $(\mathrm{DQO}=21 \mathrm{mg} / \mathrm{l})$, a pesar de que la remoción de color fuera inferior (90\%).

Por lo anterior, se recomienda escoger el tratamiento que logre la mejor remoción de materia orgánica oxidable. El tratamiento con tuna mejoró significativamente al tratamiento donde solo se aplicó sulfato de aluminio $(\mathrm{DQO}=52 \mathrm{mg} / \mathrm{l})$ en la reducción de la materia orgánica soluble (figura 3 y cuadro 3 ).

Los flóculos que forma el color son muy livianos y quebradizos y la presencia de turbidez puede darle más peso y cohesión y mejora el proceso de sedimentación (Arboleda, 2000). Por lo anterior, y considerando los resultados de la tuna en agua sintética, este producto fue evaluado como floculante en una muestra de río adicionándole solo $\mathrm{CaO}$ en I,5 mg/l.

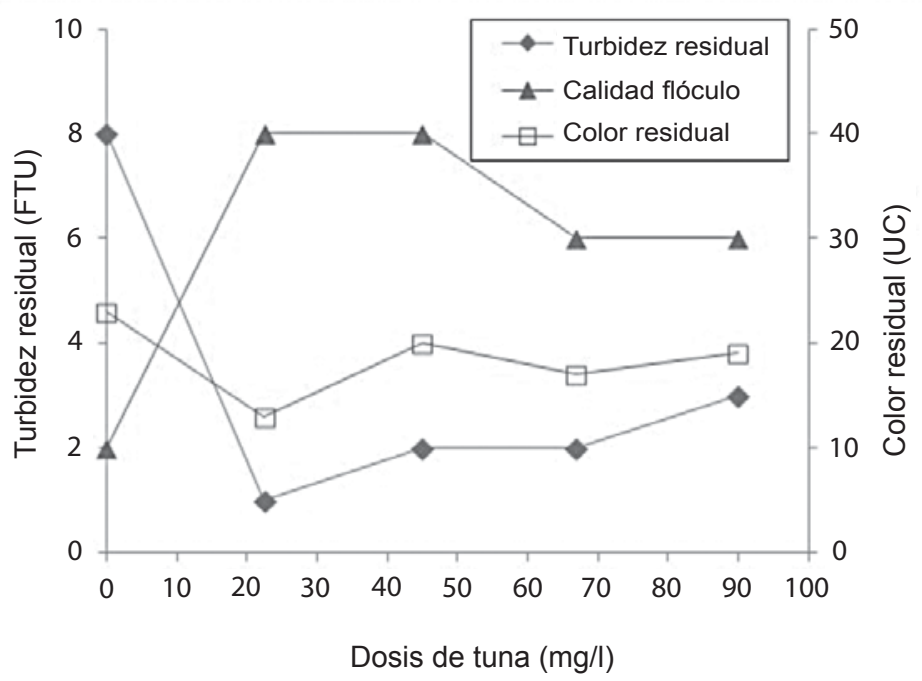

Figura 2. Color y turbidez residuales utilizando tuna como floculante en agua modelo.

Cuadro 3. Comparación de la calidad del agua artificial tratada con floculante sintético y tuna.

\begin{tabular}{|l|c|c|c|c|c|c|c|c|}
\hline \multicolumn{1}{|c|}{ Coagulante } & $\begin{array}{c}\text { Dosis } \\
\text { coagulante } \\
(\mathrm{mg} / \mathrm{l})\end{array}$ & $\begin{array}{c}\text { Dosis } \\
\text { floculante } \\
(\mathrm{mg} / \mathrm{l})\end{array}$ & $\begin{array}{c}\text { Turbidez } \\
\text { residual } \\
(\mathrm{FTU})\end{array}$ & $\begin{array}{c}\text { Color } \\
\text { residual } \\
(\mathrm{UC})\end{array}$ & $\begin{array}{c}\text { Remoción } \\
\text { turbidez } \\
(\%)\end{array}$ & $\begin{array}{c}\text { Remoción } \\
\text { color }(\%)\end{array}$ & $\begin{array}{c}\text { DQO } \\
\text { soluble } \\
(\mathrm{mg} / \mathrm{l})\end{array}$ \\
\hline $\begin{array}{l}\text { Calidad } \\
\text { flóculo }\end{array}$ \\
\hline $\mathrm{Al}_{2}\left(\mathrm{SO}_{4}\right)_{3}$ (control) & 7,0 & 0 & 8 & 23 & 60 & 86 & 52 & 2 \\
\hline $\mathrm{Al}_{2}\left(\mathrm{SO}_{4}\right)_{3}+$ Tuna & 7,0 & 22,5 & 1 & 13 & 95 & 92 & 31,5 & 8 \\
\hline $\begin{array}{l}\mathrm{Al}_{2}\left(\mathrm{SO}_{4}\right)_{3}+\text { floculante } \\
\mathrm{KF} 930-\mathrm{S}\end{array}$ & 20 & 2,0 & 2 & 16 & 90 & 89 & 10,3 & 6 \\
\hline
\end{tabular}




\section{Muestras de agua de río con $\mathrm{CaO}$}

Los parámetros de calidad del agua cruda fueron los siguientes: turbidez 17 FTU, color aparente $=138$ UC, $\mathrm{pH}=7,02$.

Los resultados muestran un mejor desempeño en la remoción del color (86\% con un valor residual de 20 UC a una dosis de tuna de I I mg/l) y aplicando dosis habituales del $(12 \mathrm{mg} / \mathrm{l})$ de sulfato de aluminio, dejando el agua tratada de conformidad con la norma (véase la figura 4).

Sin embargo, la contribución de la materia orgánica al agua filtrada (DQO $=42 \mathrm{mg} / \mathrm{l})$ fue cuatro veces superior al tratamiento con productos comerciales (véase figura 5 y cuadros 2 y 3).

Como se observa en las figuras 4 y 5 , se indica la necesidad de eliminar compuestos orgánicos sin actividad floculante de los extractos del nopal, dado que, como tendencia, la remoción del color se incrementó con la dosis del floculante pero también aumentó la materia orgánica soluble en el agua.

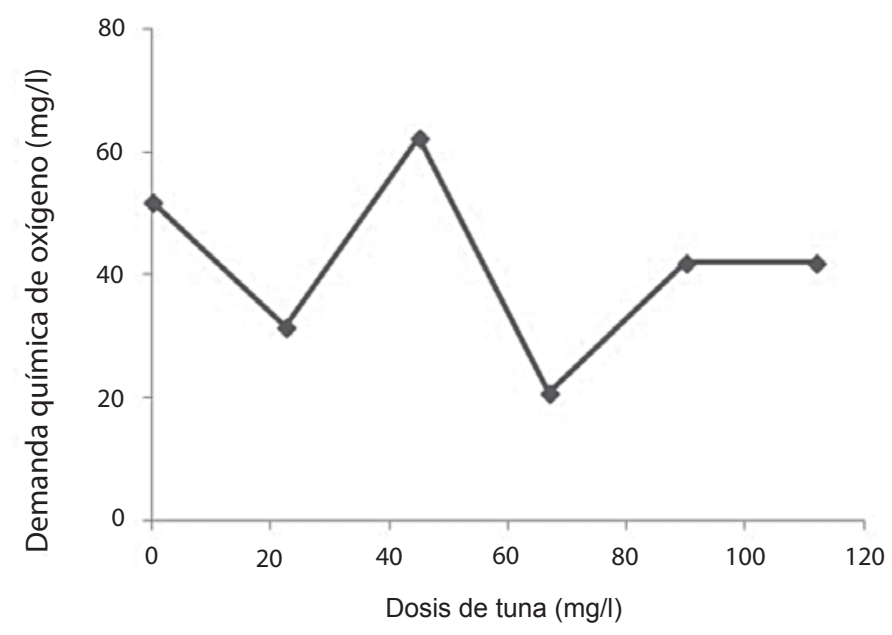

Figura 3. DQO soluble del agua tratada con tuna como floculante en agua modelo.

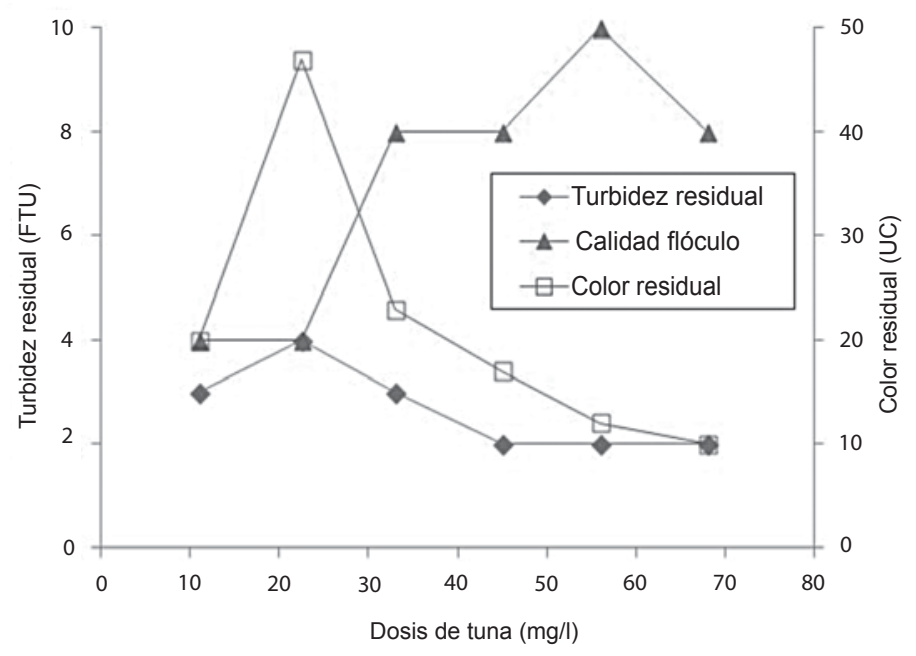

Figura 4. Color y turbidez residuales utilizando tuna como floculante en agua de río con $\mathrm{CaO}$. 


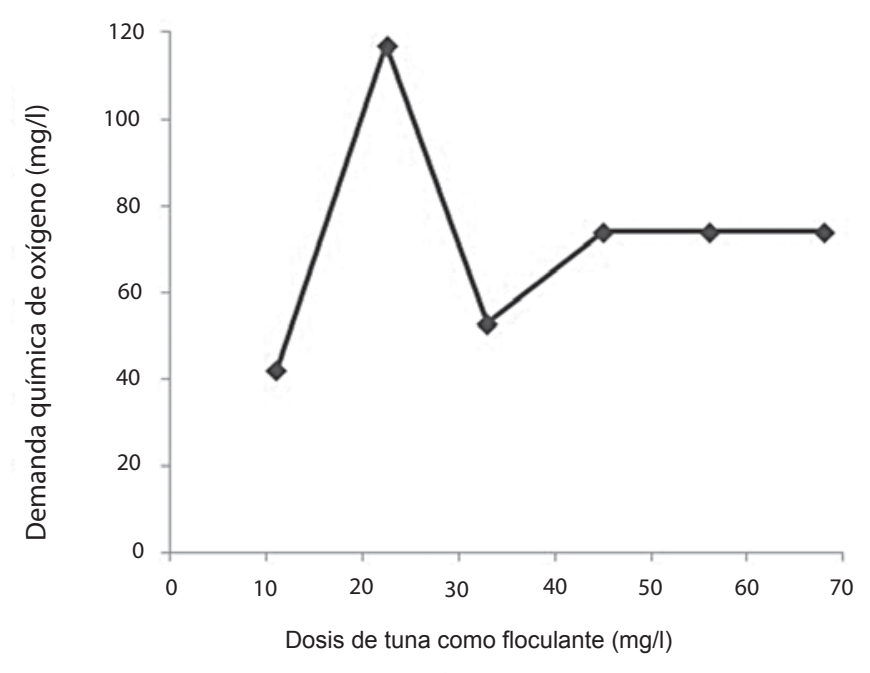

Figura 5. DQO soluble del agua tratada con tuna como floculante en agua de río con $\mathrm{CaO}$.

\section{Conclusiones}

El empleo de la tuna como coagulante o floculante para remover altos valores de color en aguas con baja turbidez, alcalinidad y dureza fue poco efectivo. Sin embargo, para el caso de aguas con mayor turbidez y alcalinidad su efectividad mejoró, incluso comparada con los productos comerciales.

Sin embargo, en el uso de este producto natural se debe considerar el aumento en la demanda de cloro, de los precursores de subproductos de desinfección y del carbono orgánico biodegradable.

Se recomienda la extracción de los ingredientes activos de la tuna para evaluar su capacidad coagulante/floculante, evitando de esta forma la carga de materia orgánica sin propiedades coagulantes.

\section{Bibliografía}

APHA, AWWA, WEP (2005). Standard Methods for the Examination of Water and Wastewater. 21 ed. USA: APHA, AWWA WEP, American Public Health Association 5-64 a 5-68.

Arboleda, J. (2000). Teoría y práctica de la purificación del agua. Tomo I, 46 pp. Bogotá: McGraw Hill.
Eikebrokk, B. (1999). Coagulation-direct filtration of soft, low alkalinity humic waters, Wat. Sci. Tech. 40: 9 , 55-62.

Gregor, J. \& Nokes, C. \& Fenton, E. ( 1997). Optimising natural organic matter removal from low turbidity waters by controlled $\mathrm{pH}$ adjustment of aluminium coagulation. Wat. Res. 3I ( I 2): 2949-2958.

Gutiérrez, F. (2009, abril 28). ICE recupera capacidad de embalse Río Macho. La Nación.

Martínez, D., Chávez, M., Díaz, A., Chacín, E. \& Fernández, N. (2003). Eficiencia de Cactus lefaria para su uso como coagulante en la clarificación de aguas. Rev. Téc. Ing. Univ. Zulia 26, I.

MINSA (2005). Compendio de Reglamentos para la calidad del Agua Potable. Vol. II, pp. I - I I.

Montero, G. (2008). Planta Potabilizadora, Tres Ríos; Instituto Costarricense de Acueductosy Alcantarillados. Com. pers.

Picado, L. 1984). Aplicación de polímeros naturales al tratamiento de potabilización del agua. Informe del Proyecto Final de Graduación, Universidad de Costa Rica.

Ruiz, R. (|99|). Identificación y remoción de color orgánico en las aguas de consumo humano. Tesis, Escuela de Química, Universidad de Costa Rica. 